\title{
Periodontitis and Chronic Obstructive Pulmonary Disease
}

\author{
Agathi Spiropoulou, Olga Lagiou, \\ Dimosthenis Lykouras, Kiriakos Karkoulias and \\ Kostas Spiropoulos
}

Additional information is available at the end of the chapter

http://dx.doi.org/10.5772/intechopen.69957

\begin{abstract}
Chronic periodontitis and chronic obstructive pulmonary disease (COPD) are chronic inflammatory diseases in which neutrophilic inflammation plays a major role. There are a few studies showing that these two entities share various predisposing factors and pathogenetic mechanisms; however, a direct connection between them has not yet been achieved. Epidemiology data may also show a connection between the two conditions. Neutrophilic inflammation in periodontitis and COPD is orchestrated by CD8+ lymphocytes and macrophages, leading to the aggregation of neutrophils and causing an imbalance to the proteases and antiproteases equilibrium. Finally, further research is needed to clarify the common pathogenesis of the two diseases to optimize their therapeutic management.
\end{abstract}

Keywords: chronic inflammation, neutrophils, oral hygiene

\section{Introduction}

Periodontitis is a chronic inflammatory disease of the oral cavity, affecting the structures that support the teeth. Almost half of the adult population has a great inflammation of the gums that causes loss of optimal contact between the teeth and periodontal tissues [1]. About $11 \%$ of adults develop clinical periodontitis. The dental plaque is caused by the development of anaerobic bacteria that cause accumulation and activation of neutrophils, which is orchestrated by various mediators and enzymes that destroy the connective tissue $[2,3]$. Untreated periodontitis ultimately leads to loss of support of the teeth, and atrophy of the alveolar process, causing loss of the teeth. 
Chronic obstructive pulmonary disease (COPD) is an inflammatory disorder of the airways, largely caused by smoking, and it is characterized by progressive and partially reversible airflow limitation [4]. The airflow obstruction and consequently the disease are confirmed by the presence of a postbronchodilator FEV1/FVC $<0.70$ [5]. The disease constitutes one of the leading causes of morbidity and mortality in the industrialized world, affecting approximately 210 million people worldwide. In 2004, COPD was the fourth most common cause of death and it is expected to be the third cause of mortality by 2030 $[6,7]$.

COPD is a combination of chronic bronchitis and emphysema, and as a result, it constitutes a heterogeneous disease. The most common symptoms in patients suffering from COPD are dyspnea, cough, and production of sputum, which are chronic and progressive [8].

The lesions present in COPD and periodontitis are linked to various immunologic mechanisms, in which T-lymphocytes, macrophages, and neutrophils have a major role. Our chapter attempts to analyze the similarities in pathophysiology of COPD and chronic periodontitis and to elucidate the common pathogenetic mechanisms.

\section{The role of chronic inflammation}

COPD and chronic periodontitis are characterized by chronic neutrophilic inflammation, which mainly stems from the activity of enzymes released from granules of neutrophils. It is known that the development of COPD depends on the variable exposure to harmful factors, such as cigarette smoking, as well as on the susceptibility of the individual [9]. It is estimated that only a percentage of smokers (5-20\%) develop COPD. Moreover, some patients develop mild disease, others moderate, and others serious disease. Among COPD patients, $80-90 \%$ have been current or ex-smokers. Air pollution and occupational exposure have also been responsible for some COPD cases at a lower rate.

On the other hand, in periodontitis, there is an interaction between environmental and genetic factors, which eventually leads to the development of the disease. In both COPD and periodontitis, the pathophysiological mechanisms include the accumulation and activation of neutrophils. Factors secreted by neutrophil granules cause damage to the connective tissue [10].

It is believed that COPD is a condition of chronic systemic inflammation, characterized by elevated C-reactive protein (CRP), interleukin-8 (IL-8), and tumor necrosis factor- $\alpha$ (TNF- $\alpha$ ), whose levels determine the severity of disease, which is in line with the degree of muscle atrophy and dysfunction. These cytokines are also related to the development of coronary artery disease and diabetes [11]. Morbidity and mortality also depend on social and economic factors [12].

Inflammatory responses taking place in periodontitis are usually caused by the presence of anaerobic microbes. Thus, the levels of pro-inflammatory cytokines, such as CRP and TNF- $\alpha$, 
are remarkably elevated. In both COPD and periodontitis, there is an increased incidence of heart attack, osteoporosis, diabetes mellitus and rheumatoid arthritis as a result of chronic inflammation [13].

\section{Epidemiology of COPD and periodontitis}

A variety of stimuli can induce the development of COPD, including heritable genes in conjunction with environmental risk factors. Air pollutants, cold temperature, lack of compliance with respiratory medication, and other noninfectious causes, as well as infections, are the usual triggers of acute exacerbations of COPD. Infections, which are the most frequent cause of exacerbations, could be of either bacterial or viral etiology [14]. It is known that almost half of the infections are caused by bacteria, while viruses are responsible for almost the rest of the infections. Moreover, coinfection with bacteria and viruses is identified in patients with severe COPD.

Smoking is an important predisposing factor both in COPD and in periodontitis. Almost $80 \%$ of COPD patients are current or ex-smokers. COPD is also associated with age, impaired lung function, and gender. Initially, it was believed that the impact was greater in men, but more recent data exhibit equal or greater sensitivity of women smokers to develop COPD [15].

A working environment involving exposure to dust and harmful gases increases the risk of developing the disease. When smoking coexists, there is a sixfold risk. Exposure to inhaled gases from burning biomass increases the risk. There are not causative microbes causing COPD to develop. However, several viruses and bacteria are responsible for COPD disease exacerbations that lead to impaired lung function and quality of life deterioration [16].

Smoking is also a risk factor for the development of periodontitis, and the severity of the disease depends on the density of smoking habit [17]. Men and elderly people are more susceptible for COPD and periodontitis. Other predisposing factors include diabetes and poor socioeconomic conditions. Since 1990, there has been an increasing interest in the possible links between COPD and periodontitis. There is a correlation between poor oral hygiene and COPD [18].

In a study of military veterans, a diagnosis of COPD was made in subjects with an FEV1/FVC ratio $<70 \%$ and a history of smoking. The existence of periodontitis was documented by $\mathrm{X}$-ray results which show the loss of the alveolar bone. It was observed that a loss of $20 \%$ of the alveolar bone leads to an increased risk for development of COPD by $60 \%$. However, it should be noted that the reduction of FEV1 is related not only to the existence of COPD but also to other respiratory diseases [19].

Other notable studies attempt to correlate COPD severity with the existence of periodontitis using more comprehensive definitions for the existence of COPD. In a study of 600 people with COPD was shown that the risk of developing COPD was associated directly with the quality of oral hygiene [20]. More specifically, patients with COPD had a higher dental plaque index, and an insufficient support of the tooth from the surrounding tissues. Moreover, the same patients 
had an unsatisfactory behavior regarding their oral hygiene, as measured by the frequency of toothbrushing, the use of dental yarns, and the frequency of visits to the dentist [21]. In another study, a direct correlation between COPD severity and poor hygiene was found in general. Therefore, this observation implies that the possibility of poor oral hygiene and the unfavorable course of COPD may be linked to nonhealthy lifestyle and poor socioeconomic conditions [22].

It is known that the pathogenesis of COPD is directly associated with the pathological relationship of proteases/antiproteases in the lung. The same theory has been suggested in periodontitis. Past studies have shown that in patients suffering from mild COPD and periodontitis the levels of metalloproteinase-8 (MMP-8) are elevated in saliva and serum. MMP-8 is a product of the secretion of neutrophils. However, levels of MMP-8 in saliva showed no statistically significant difference in patients with mild COPD who had no periodontitis compared to those that did not suffer from COPD. Some other studies demonstrate a poor correlation between COPD and periodontitis [23].

To sum up, several meta-analyses have shown that periodontitis increases the risk of developing COPD; however, the exact mechanism is still not fully understood. Future studies should focus on the elucidation of such pathogenetic mechanisms.

\section{Pathophysiology of COPD and periodontitis}

COPD is characterized by limitation of airflow which is partially reversible. Progressive decline of FEV1, inadequate lung emptying on expiration, and static and dynamic hyperinflation are the results of remodeling of the small-airway compartment and loss of elastic recoil by emphysematous destruction of parenchyma [24]. Exposure to smoke leads to infiltration of the mucosa, submucosa, and glandular tissue by inflammation cells. Increased mucus content, epithelial-cell hyperplasia, and disturbed tissue repair with wall thickening in the small conducting airways are the main features of COPD [25].

Smoking can cause injury of airway epithelial cells, and as a result, endogenous signals are released and recognized by receptors such as Toll-like receptors 4 and 2 on epithelial cells. This recognition leads to a nonspecific inflammatory response which involves the release of early cytokines, macrophages, neutrophils, and dendritic cells and the transportation of these features to the site of inflammation [26]. Self-antigens released from damaged tissue as well as foreign antigens from incoming pathogens are presented to naïve T-cells by dendritic cells. The T-cells are activated into T-helper-1 cells, and these specific CD4 and CD8 cells as well as B-cells which produce antibodies are transferred to the lungs so as to neutralize the antigens [27].

Alveolar macrophages and other immune cells produce proteinases that destroy the basal membrane and also cause damage to the collagen and elastic fibers of the connective tissue, leading to the development of emphysema [28]. Moreover, they secrete IL-4 and IL-3 that cause edema and increased production of mucus, which are both associated with airway hyperresponsiveness. These responses take place at the respiratory lobule of second order that is located after the terminal bronchiole. It consists of 3-5 generations of respiratory bronchioles that contain 
alveoli. This is the structure that is responsible for the exchange of respiratory gases, and its destruction may cause impaired gas exchange and respiratory failure [29]. Moreover, apart from the lack of $\alpha-1$ antitrypsin, other genetic factors involved in the pathogenesis of the disease include interleukins and cysteine proteinases and elastases, which orchestrate an immune response which can lead to the destruction of the pulmonary parenchyma. Mast cells play an important role as antigen-presenting cells in the lung as well, and they have been shown to be valuable both in COPD and in periodontitis.

Smoking is the main factor that initiates immune system reaction in chronic inflammatory diseases as the chronic obstructive pulmonary disease and periodontitis [30]. In response to smoking, neutrophils accumulate rapidly in the lung, because the macrophages and epithelial cells of the lung are activated. They secrete neutrophil-attracting factors such as IL-8, C5a, and LTB4. The stimulation of neutrophils causes oxidative damage to the lung due to oxygen radicals produced by activated neutrophils. Moreover, free oxygen radicals cause oxidative injury to the lung [31]. Oxygen radicals play a role in premature cellular death, but also act on the epithelial and mesenchymal cells. The destruction of the cellular matrix causes a destruction of the supporting connective tissue. Some of these polypeptides as laminin and fibronectin have chemotactic effect and attract neutrophils, playing a key role in the sequence of events of the destruction of lung parenchyma due to smoking. Neutrophils contain proteinases stored in their granules, which are released and destroy the parenchyma. There are metalloproteinase- 9 (MMP-9) and serine proteinases, which are released and destroy elastin. Elastin is the characteristic component of elastic fibers, which determine the elastic properties of the lung parenchyma. The destruction of the elastic fibers causes an increased lung compliance and reduction of elasticity, which are characteristic changes in pulmonary emphysema [32]. Elastin segments act as chemotactic agents for macrophages. The aggregated macrophages secrete metalloproteinases in turn and participate in the destruction of lung parenchyma. Macrophages secrete chemokines, which maintain the chronic inflammation that characterizes COPD (Figure 1) [33].

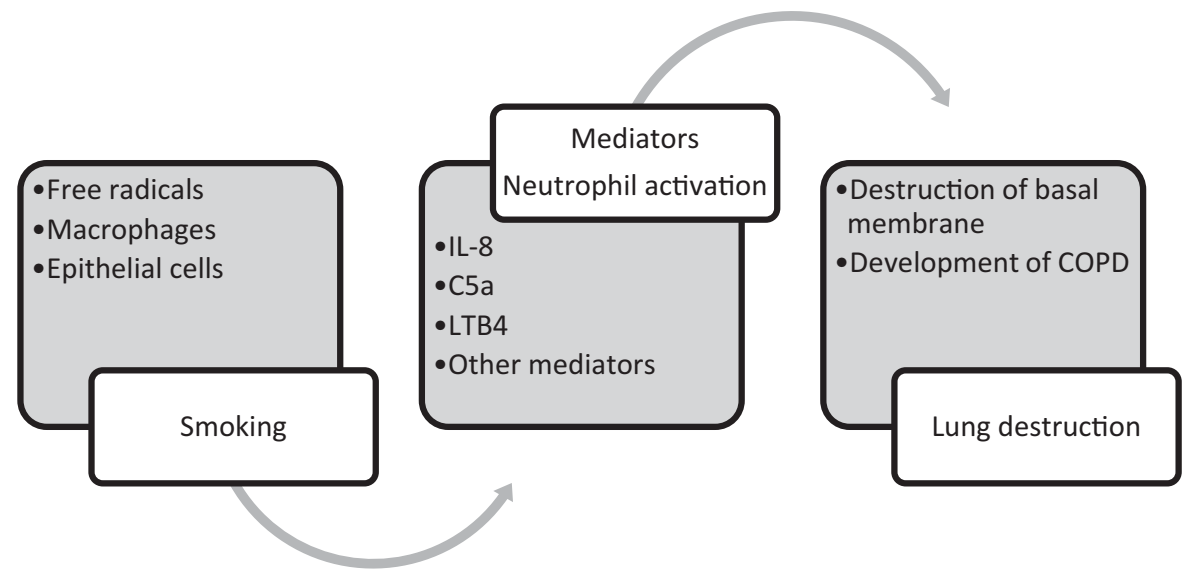

Figure 1. Pathogenetic mechanisms of lung destruction in COPD. 
Apart from neutrophils and macrophages, there are also increased numbers of CD4+ T lymphocytes and CD8+ T lymphocytes in bronchioles and alveoli of COPD patients. Epithelial cells of smokers with COPD show an increased expression of CXC40 factor, which is a structural element of the agent of T-cell, CXRCR3 [34]. Although the precise role of T cells is not fully understood, it seems that they produce metalloproteinases, which have profound destructive effects on pulmonary parenchyma. The cytotoxic T-cells are also likely to affect epithelial cells, leading to premature cellular death. Other cells, such as dendritic cells and eosinophils, have also been reported to be increased in COPD. But their exact role is still unknown.

Cigarette smoking, as previously explained, is the main factor to initiate chronic inflammation. However, a few other factors cause inflammation to perpetuate, even years after smoking cessation. Indeed, in histological lung preparations derived from pneumonectomy, inflammation cells, such as macrophages, T-cells, neutrophils, and eosinophils are observed, even 9 years after the end of smoking habit [35]. The exact mechanism of persistent inflammation is not known, but it seems that the impaired mobility of the cilia of epithelial cells of the respiratory mucosa predisposes to the colonization of airways with bacteria, which in turn predisposes to viral infections, especially adenovirus infections, which trigger the inflammatory processes. Despite the controversial pathogenesis, numerous inflammatory cells participate in COPD pathogenesis and cause inflammation that proceeds even when the original trigger of cigarette smoke is absent.

These pathogenetic mechanisms have not yet been confirmed in the case of periodontitis. It is known that neutrophils are the inflammatory cells that predominate in cases of gingivitis [36] and their role in the pathogenesis of chronic periodontitis has been investigated. But, it is not yet fully understood whether the neutrophils have the same characteristics and the same role in both periodontitis and COPD. Further research is needed in the field. However, it is known that in both COPD and periodontitis, activated neutrophils secrete inflammatory mediators, such as proteinases and oxygen radicals, which are involved in tissue damage and in chronic inflammation. This is an important feature of both conditions. Moreover, increased free radicals are produced when cells are exogenously stimulated by the means of Fusobacterium nucleatum, a bacterium that frequently causes periodontitis [37].

It should be noted that adverse effects are also caused when local levels of antioxidant factors in interstitial fluid gum are disrupted. At this point, we should remark that if the hypothesis of diffuse inflammation is adopted, we can assume that successful treatment of one disease (periodontitis) could improve the other (COPD) and conversely. It is known that there is a positive correlation between poor oral hygiene and the frequency of COPD exacerbations [38]. In a recent study, patients with COPD and concomitant periodontitis were tested. Half of them (20 patients) received proper treatment for periodontitis, while the rest 20 patients did not receive any treatment at all. They were all observed for 12 months, and it was reported that in the first group a lower frequency of COPD exacerbations was documented. Although this is a significant finding, the study had some methodological problems such as the fact that the selection of patients was not randomized and the sample size was small. 
Nevertheless, the findings of this study should be carefully considered in order to carry out further investigation in the field.

\section{Antibiotic treatment in chronic periodontitis and COPD}

Exacerbations are short periods in the course of COPD characterized by increased cough, dyspnea, and production of sputum that can become purulent. They can lead to accelerated lung function impairment, worse quality of life, and increased mortality [39]. Except from the severity, the frequency of the exacerbations, also, plays an important role in the management of the exacerbations. Depending on the severity of the exacerbation, different therapeutic strategies could be used. Therefore, increased doses of bronchodilators are required for mild exacerbations, systemic corticosteroids, and antibiotics can be used for moderate exacerbations, whereas severe exacerbations often require admission to hospital [40]. However, the most frequent causes of exacerbations are bacterial or viral infections, which are responsible for $60-80 \%$ of all exacerbations. These infectious exacerbations are more severe than the noninfectious exacerbations.

There are some articles concerning the possible relationship between chronic inflammatory diseases and their comorbidities. Both chronic periodontitis and COPD are neutrophilic, inflammatory conditions characterized by loss of local connective tissue. It is possible that there is an association and perhaps a casual link between the two diseases. It has been reported that respiratory pathogens such as Pseudomonas aeruginosa might adhere better to oral epithelial cells obtained from patients colonized by respiratory pathogens than to cells harvested from noncolonized patients. Trypsin treatment of epithelial cells from noncolonized patients in vitro resulted in increased adhesion of respiratory pathogens [41]. This suggests that mucosal alteration promoted enhanced bacterial adhesion of these bacteria. This alteration is perhaps the loss of fibronectin (by exposure to proteases) from the epithelial cell surface, which may unmask mucosal surface receptors for respiratory pathogen adhesions.

Subjects with poor hygiene may have elevated levels of hydrolytic enzymes in their saliva. These enzymes may process mucins to reduce their ability to bind to and clear pathogens such as Haemophilus influenzae. Conversely, the enzymes may process the respiratory epithelium to modulate the adhesion of such pathogens to the mucosal surface. Mannino et al. [42] postulated that oral pathogens continuously stimulate the cells of the periodontium (epithelial cells) to release a wide variety of cytokines and other biologically active molecules such as IL-1a, IL-1b, IL-6, IL-8, and TNF-a. Oral bacteria in secretions may adhere to the mucosal surface and stimulate the epithelial cells of the respiratory epithelium to secrete cytokines. These stimulated cells may then release other cytokines that recruit inflammatory cells to the site. These inflammatory cells release hydrolytic enzymes resulting in damaged epithelium that may be more susceptible to colonization by respiratory pathogens. It is possible that the poor oral health might work in concert with other factors (smoking, environmental pollution, viral infections, and genetic factors) to promote the progression and exacerbation of pulmonary disease. 


\section{Conclusions}

The associations demonstrated between periodontitis and COPD suggest a basis for testing the effects of treatment for one condition upon the severity of the other. Improving oral hygiene might reduce the risk of respiratory infection among subjects who are at risk.

Our article describes similarities in epidemiology and pathogenetic mechanisms of COPD and periodontitis and proposes that improvement of one condition is linked to the treatment of the other. Future research is needed to clarify the existing pathogenetic mechanisms and extend possible therapeutic options.

\section{Authors' contributions}

Agathi Spiropoulou and Dimosthenis Lykouras have worked on manuscript concept, literature review, and manuscript preparation. Olga Lagiou worked on literature review and manuscript preparation. Kiriakos Karkoulias worked on manuscript preparation. Kostas Spiropoulos worked on revision of manuscript for important intellectual content.

\section{Abbreviations}

$\begin{array}{ll}\text { COPD } & \text { Chronic obstructive pulmonary disease } \\ \text { CD } & \text { Cluster of differentiation } \\ \text { CRP } & \text { C-reactive protein } \\ \text { MMP } & \text { Metalloproteinase } \\ \text { IL } & \text { Interleukin } \\ \text { TNF } & \text { Tumor necrosis factor } \\ \text { (FEV1/FVC) ratio } & \begin{array}{l}\text { The ratio of the forced expiratory volume in the first one second to } \\ \text { the forced vital capacity of the lungs }\end{array} \\ \text { LTB4 } & \text { Leukotriene B4 } \\ \text { C5a } & \text { Complement component 5a }\end{array}$

\section{Author details}

Agathi Spiropoulou, Olga Lagiou, Dimosthenis Lykouras, Kiriakos Karkoulias and Kostas Spiropoulos*

*Address all correspondence to: spircos@upatras.gr

Department of Pulmonary Medicine, University Hospital of Patras, Rio, Patras, Greece 


\section{References}

[1] Morris AJ, Steele J, White DA. The oral cleanliness and periodontal health of UK adults in 1998. British Dental Journal. 2001;191:186-192

[2] Kinane DF, Preshaw PM, Loos BG, Working Group 2 of Seventh European Workshop on Periodontology. Host-response: Understanding the cellular and molecular mechanisms of host-microbial interactions - Consensus of the Seventh European Workshop on Periodontology. Journal of Clinical Periodontology. 2011;38(Suppl 11):44-48

[3] Listgarten MA. Pathogenesis of periodontitis. Journal of Clinical Periodontology. 1986;13:418-430

[4] Sampsonas E, Lykouras D, Drakatos P, Moschopoulou A, Spiropoulos K, Karkoulias K. Endothelin-1 polymorphisms involved in impaired exercise tolerance in COPD patients. A pilot study. European Review for Medical and Pharmacological Sciences. 2011;15(2):123-128

[5] Rabe KF, Hurd S, Anzueto A, et al. Global strategy for the diagnosis, management, and prevention of chronic obstructive pulmonary disease. American Journal of Respiratory and Critical Care Medicine. 2007;176(6):532-555

[6] GOLD. Global Strategy for the Diagnosis, Management and Prevention of COPD. Global Initiative for Chronic Obstructive Lung Disease (GOLD) [Online]. 2014. Available from: http://www.goldcopd.org/uploads/users/files/GOLD_Report_2014_Jun11.pdf

[7] Lopez AD, Mathers CD, Ezzati M, Jamison DT, Murray CJ. Global and regional burden of disease and risk factors, 2001: Systematic analysis of population health data. Lancet. 2011;367:1747-1757

[8] Tzortzaki E, Siafakas N. Paolo Palange, Anita K. Simonds. COPD and emphysema. In: ERS Handbook: Respiratory Medicine. 2nd ed. European Respiratory Society. UK; 2013. pp. 287-292. ISBN: 978-1-84984-040-8

[9] Stockley RA. Neutrophils and the pathogenesis of COPD. Chest. 2002;121(Suppl 5): 151S-155S

[10] Saetta M. Airway inflammation in chronic obstructive pulmonary disease. American Journal of Respiratory and Critical Care Medicine. 1999;160:S17-S20

[11] Barnes PJ, Celli BR. Systemic manifestations and comorbidities of COPD. European Respiratory Journal. 2009;33:1165-1185

[12] Sherrill DL, Lebowitz MD, Burrows B. Epidemiology of chronic obstructive pulmonary disease. Clinics in Chest Medicine. 1990;11:375-387

[13] Lee HJ, Garcia RI, Janket SJ, Jones JA, Mascarenhas AK, Scott TE, et al. The association between cumulative periodontal disease and stroke history in older adults. Journal of Periodontology. 2006;77:1744-1754

[14] Wedzicha J, Seemungal T. COPD exacerbations: Defining their cause and prevention. Lancet. 2007;370:786-796 
[15] Connett JE, Murray RP, Buist AS, Wise RA, Bailey WC, Lindgren PG, et al. Changes in smoking status affect women more than men: Results of the Lung Health Study. American Journal of Epidemiology. 2003;157:973-979

[16] Mannino DM. COPD: Epidemiology, prevalence, morbidity and mortality, and disease heterogeneity. Chest. 2002;121(Suppl 5):121S-126S

[17] Pihlstrom BL. Periodontal risk assessment, diagnosis and treatment planning. Periodontal 2000. 2001;25:37-58

[18] Scannapieco FA, Papandonatos GD, Dunford RG. Associations between oral conditions and respiratory disease in a national sample survey population. Annals of Periodontology. 1998;3:251-256

[19] Deo V, Bhongade M, Ansari S, Chavan RS. Periodontitis as a potential risk factor for chronic obstructive pulmonary disease: A retrospective study. Indian Journal of Dental Research. 2009;20:466-470

[20] Zhou X, Wang Z, Song Y, Zhang J, Wang C. Periodontal health and quality of life in patients with chronic obstructive pulmonary disease. Respiratory Medicine. 2011;105:67-73

[21] Wang Z, Zhou X, Zhang J, Zhang L, Song Y, Hu FB, et al. Periodontal health, oral health behaviours, and chronic obstructive pulmonary disease. Journal of Clinical Periodontology. 2009;36:750-755

[22] Liu Z, Zhang W, Zhang J, Zhou X, Zhang L, Song Y, et al. Oral hygiene, periodontal health and chronic obstructive pulmonary disease exacerbations. Journal of Clinical Periodontology. 2012;39:45-52

[23] Yildirim E, Kormi I, Basoğlu ÖK, Gürgün A, Kaval B, Sorsa T, et al. Periodontal health and serum, saliva matrix metalloproteinases in patients with mild chronic obstructive pulmonary disease. Journal of Periodontal Research. 2013;48:269-275

[24] O'Donnell DE. Hyperinflation, dyspnea, and exercise intolerance in chronic obstructive pulmonary disease. Proceedings of the American Thoracic Society. 2006;3:180-184

[25] Hogg JC, Timens W. The pathology of chronic obstructive pulmonary disease. Annual Review of Pathology. 2009;4:435-459

[26] Barnes PJ, Shapiro SD, Pauwels RA. Chronic obstructive pulmonary disease: Molecular and cellular mechanisms. European Respiratory Journal. 2003;22:672-688

[27] Rahman I, Adcock IM. Oxidative stress and redox regulation of lung inflammation in COPD. European Respiratory Journal. 2006;28:219-242

[28] Shapiro SD. The macrophage in chronic obstructive pulmonary disease. American Journal of Respiratory and Critical Care Medicine. 1999;160:S29-S32

[29] Kheradmand F, Shan M, Xu C, Corry DB. Autoimmunity in chronic obstructive pulmonary disease: Clinical and experimental evidence. Expert Review of Clinical Immunology. 2012;8:285-292 
[30] Ali J, Pramod K, Tahir MA, Ansari SH. Autoimmune responses in periodontal diseases. Autoimmunity Reviews. 2011;10:426-431

[31] Krinsky NI. Mechanism of action of biological antioxidants. Proceedings of the Society for Experimental Biology and Medicine. 1992;200:248-254

[32] Thurlbeck W. Pathology of chronic airflow obstruction. In: Cherniack NS, editor. Chronic Obstructive Pulmonary Diseases. Philadelphia: WB Saunders; 1991. pp. 3-20

[33] Silverman EK, Chapman HA, Dreazen JM, Weiss ST, Rosner B, Campbell EJ, et al. Genetic epidemiology of severe, early-onset chronic obstructive pulmonary disease: Risk to relatives for airflow obstruction and chronic bronchitis. American Journal of Respiratory and Critical Care Medicine. 1998;157:1770-1778

[34] Rennard SI. Repair. In: Calverley PMA, MacNee W, Pride NB, Rennard SI, editors. Chronic Obstructive Pulmonary Disease. London: Arnold; 2003. pp. 139-150

[35] Saetta M, Di Stefano A, Turato G, Facchini FM, Corbino L, Mapp CE, et al. CD8+ T-lymphocytes in peripheral airways of smokers with chronic obstructive pulmonary disease. American Journal of Respiratory and Critical Care Medicine. 1998;157:822-826

[36] Matthews JB, Wright HJ, Roberts A, Cooper PR, Chapple IL. Hyperactivity and reactivity of peripheral blood neutrophils in chronic periodontitis. Clinical \& Experimental Immunology. 2007;147:255-264

[37] Nussbaum G, Shapira L. How has neutrophil research improved our understanding of periodontal pathogenesis? Journal of Clinical Periodontology. 2011;38(Suppl 11):49-59

[38] Kucukcoskun M, Baser U, Oztekin G, Kiyan E, Yalcin F. Initial periodontal treatment for prevention of chronic obstructive pulmonary disease exacerbations. Journal of Periodontology. 2012;84:863-870

[39] Llor C, Moragas A, Hernandez S, Bayona C, Miravitlles M. Efficacy of antibiotic therapy for acute exacerbations of mild to moderate chronic obstructive pulmonary disease. American Journal of Respiratory and Critical Care Medicine. 2012;186:716-723

[40] Decramer M, Janssens W, Miravitlles M. Chronic obstructive pulmonary disease. Lancet. 2012;379:1341-1351

[41] Zandvoort A, van der Geld YM, Jonker MR, Noordhoek JA, Vos JT, et al. High ICAM-1 gene expression in pulmonary fibroblasts of COPD patients: A reflection of an enhanced immunological function. European Respiratory Journal. 2006;28:113-122

[42] Mannino DM, Doherty DE, Sonia Buist A. Global Initiative on Obstructive Lung Disease (GOLD) classification of lung disease and mortality: Findings from the Atherosclerosis Risk in Communities (ARIC) study. Respiratory Medicine. 2006;100:115-122 
